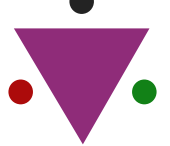

IJCRR

Section: Healthcare

Sci. Journal Impact

Factor: $6.1(2018)$

ICV: 90.90 (2018)

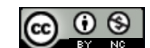

Copyright@IJCRR

\section{Neuroprotective Activity of Pyrazolone Derivatives Against Paraquat-induced Oxidative Stress and Locomotor Impairment in Drosophila melanogaster}

\author{
Sindhu Priya E S ${ }^{1,2}$, Roopa P Nayak ${ }^{3}$, Prema Saldanha ${ }^{4}$, Mohan B j5, \\ Ashwini Prabhu²
}

\begin{abstract}
'Assistant Professor, Yenepoya Pharmacy College and Research Centre, Yenepoya (Deemed to be University), University Road, Deralakatte, Mangalore-575018, India; ${ }^{2}$ Assistant Professor, Yenepoya Research Centre, Yenepoya (Deemed to be University), University Road,

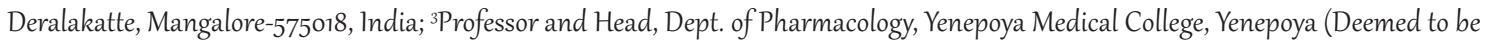

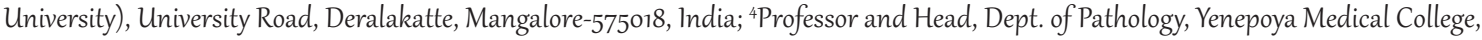
Yenepoya (Deemed to be University), University Road, Deralakatte, Mangalore-575018, lndia; 5 Research scholar, Dept of Chemistry, P A College of Engineering, Nadupadavu, Mangalore-574153, India.
\end{abstract}

\title{
ABSTRACT
}

Objectives: In the present study, we have demonstrated the antioxidant and neuroprotective effects of certain substituted hydrazine pyrazolones.

Methods: The compounds were synthesized and characterized by spectral data by reported methods. The antioxidant ability of the compounds was confirmed through in vitro antioxidant (DPPH scavenging, ABTS radical scavenging, total antioxidant capacity, and ferric reducing activity) studies. In vivo neuroprotective activity of the test, compounds were determined in Drosophila melanogaster Oregon K (OK) adult male flies. Oxidative stress was induced by using paraquat (PQ). Edaravone (EDA) was used as a standard for studies.

Results: Compound C4 was efficient in the modulation of lipid peroxidation marker levels such as malondialdehyde (MDA) and hydroperoxide (HP). Glutathione (GSH) levels were elevated in C4 treated flies significantly. The modulatory effect on enzyme antioxidants superoxide dismutase (SOD) and catalase (CAT) was observed in compound pretreatedflies. Pretreatment of compounds through dietary feeding and co-exposure to $P Q$ showed a lower death rate in flies. The neuroprotective efficacy of the compounds was confirmed through a negative geotaxis assay.

Conclusion: Flies treated with compounds and PQ co-exposure showed improvement in motor activities, suggesting the neuroprotective potential of pyrazolone derivatives. This might be due to their antioxidant ability while the precise mechanism of action needs further investigations. Among the tested compounds, C4 showed significant antioxidant and neuroprotective activity.

Key Words: Neuroprotection, Drosophila, Oxidative stress, Pyrazolone, Paraquat, Antioxidant

\section{INTRODUCTION}

Oxidative stress could play a vital role in many neurological disorders, such as epilepsy, Alzheimer's, Parkinsons disease, stroke, cerebral ischemia, multiple sclerosis, Huntington's chorea, tardive dyskinesia, amyotrophic lateral sclerosis, and other diseases. ${ }^{1}$ Paraquat (PQ) has been used as a broad-spectrum herbicide which is extremely toxic and leads to multiple organ dysfunction in humans. This pesticide was used as a model factor inducing oxidative stress in vivo mainly hydro- gen peroxide and hydroxyl radical. ${ }^{2,3}$ It has been shown that PQ causes damage to the number of dopaminergic neurons due to its ability to penetrate the blood-brain barrier (BBB). ${ }^{4}$ Drosophila melanogaster was used in this study because it has been an excellent model for gerontological research due to its relatively short life span and also appears to show many of the manifestations of cellular senescence observed in mammals. Oxidative stress plays an important role in governing the life span of the fly. ${ }^{5}$ Pyrazolone scaffold possesses

\section{Corresponding Author:}

Dr. Roopa P Nayak, Department of Pharmacology, Professor and Head, Yenepoya Medical College, Yenepoya (Deemed to be University), Mangaluru-575018; E-mail: roopapnayak@yenepoya.edu.in

ISSN: 2231-2196 (Print)

Received: 08.08 .2020
ISSN: $0975-5241$ (Online)

Revised: 23.09 .2020
Accepted: 20.10 .2020
Published: 07.12 .2020 
a broad spectrum of applications including anti-inflammatory, antioxidant, neuroprotective, anticonvulsant, and antidepressant activity. ${ }^{6,7}$

Many commercially available pyrazolone derivatives are in use for the treatment of various neurological diseases. Edaravone (EDA), a pyrazolone derivative was also used in the treatment of acute ischemic stroke and is used as a standard drug in this study. ${ }^{8,9}$ This work was attributed to the evaluation of neuroprotective efficacy of some reported edaravone derivatives in the D.melanogaster Oregon-K(OK) flies model system through the antioxidant mechanism and negative geotaxis assay.

\section{MATERIALS AND METHODS}

The analytical grade chemicals and the reagents required for the study were procured from Sigma and Himedia Pvt Ltd., India.

\section{Synthesis of pyrazolone derivatives}

The compounds (4E)-4-[2-(4-fluorophenyl) hydrazinylidene]- 5-methyl-2,4-dihydro-3H-pyrazol-3-one (C1), (4E)-4-[2-(3-chloro-4-fluorophenyl)hydrazinylidene]5-methyl-2,4-dihydro-3H-pyrazol-3-one (C2) and 3-Methyl 5-Pyrazolone (C4) were synthesized and characterized by reported methods. ${ }^{10,11}$ The structures of the compounds were further characterized by recording FT-IR, ${ }^{1} \mathrm{H} \&{ }^{13} \mathrm{C}$ NMR, and LC-MS data (Data not given). The melting point was recorded on a melting point apparatus taken in an open capillary tube and was uncorrected. The purity of the compound was confirmed by thin-layer chromatography using Merck silica gel 60 F254 coated aluminium plates. The IR spectrum was recorded on a Shimadzu-FT-IR Infrared spectrometer in $\mathrm{KBr}\left(\mathrm{Vmax}\right.$ in $\mathrm{cm}^{-1}$ ) (Shimadzu, Kyoto, Japan). The ${ }^{1} \mathrm{H}-$ NMR (400 MHz) spectrum was recorded on a Bruker AMX 400 spectrometer (Bruker Optik, Ettlingen, Germany), with $5 \mathrm{~mm}$ PABBO BB-1H TUBES at IISC, Bengaluru, Karnataka, India. The ${ }^{13} \mathrm{C}-\mathrm{NMR}(100 \mathrm{MHz})$ spectrum was recorded on a Bruker Ascend for approximately $0.03 \mathrm{M}$ solutions In $\mathrm{DMSO}_{6}$, at IISC, Bengaluru. The mass spectrum was obtained using Agilent 1200 series LC and Micromass ZQ spectrometer and a Shimadzu LCMS-8030 mass spectrometer (Shimadzu Corporation, Kyoto, Japan), operating at 70 $\mathrm{eV}$ at Mangalore University, Managalagangothri, Karnataka, India. The elemental analysis was carried out using a CHN$\mathrm{SO}$ analyser.

\section{In vitro antioxidant studies}

All the compounds were determined for in vitro antioxidant efficacy at $100 \mu \mathrm{g} / \mathrm{ml}$ concentration.DPPH radical scavenging assay was carried and data compared with vitamin $\mathrm{C}$ (Ascorbic acid -AA) and $\mathrm{IC}_{50}$ for synthesized compounds were reported. ${ }^{12} \mathrm{ABTS}$ radical scavenging activity was performed using Trolox as standard. Results are expressed as Trolox equivalents in mean \pm standard deviation. ${ }^{13}$ Total Antioxidant Capacity assay was performed using ascorbic acid as standard and results were expressed as mg Ascorbic Acid Equivalent (AAE) of the test compound in mean \pm standard deviation. ${ }^{14}$ Ferric reducing antioxidant power assay was performed using ascorbic acid as a reference standard and results were expressed as AAE of the test compound in mean \pm standard deviation. ${ }^{15}$

\section{Drosophilaculture and compound treatment}

D. melanogaster (Oregon K) adult males (8-10 days old) were obtained from Drosophila stock centre, Department of Studies in Zoology, University of Mysore, Manasagangotri, Mysore, Karnataka, India. The flies were maintained at constant temperature and humidity $\left(22 \pm 1^{\circ} \mathrm{C}\right.$ and $60-70$ $\%)$, fed on a standard wheat cream agar medium seeded with yeast. ${ }^{16,17}$ The test compounds $\mathrm{C} 1, \mathrm{C} 2, \mathrm{C} 4$, and standard EDA were dissolved in $1 \%$ dimethyl sulfoxide (DMSO). The compounds were introduced into the medium at semisolid state, mixed well and was allowed to solidify. 50 adult males were introduced into the vials containing media for further studies. All the compounds were evaluated for their toxicity by the lethality test. The male flies were fed on a medium containing $\mathrm{C} 1, \mathrm{C} 2$, and $\mathrm{C} 4$ at 50,100 and $200 \mu \mathrm{g} / \mathrm{ml}$ concentrations. Lethality due to compounds at all concentrations and solvent was monitored by counting dead flies every $24 \mathrm{~h}$ up to 7 days, and data was expressed in terms of percentage mortality. The experiment was conducted in triplicates. ${ }^{17}$

\section{Paraquat exposure studies}

In a preliminary study, flies were exposed to paraquat at concentrations of $10,15,20$ and $25 \mathrm{mM}$ for $96 \mathrm{~h}$ to determine lethality concentration due to paraquat at experimental concentration. The results were expressed as percent mortality. However, C1, C2, C4 and $15 \mathrm{mM}$ concentrations of paraquat was employed to assess the antioxidant effects of test compounds against paraquat-induced lethality. In this assay, PQ exposed flies were fed with C1, C2, C4 and EDA (200 $\mu \mathrm{g} /$ $\mathrm{ml}$ ) in the diet (co-exposure). Lethality of the flies was monitored for $96 \mathrm{~h}$ and data was expressed in terms of percent survival. ${ }^{17}$

\section{Study design for compound treatment}

1. Group I: Wild type flies fed with conventional media with $200 \mu \mathrm{L} 1 \%$ DMSO

2. Group II: Wild type flies fed with media containing paraquat

3. Group III: Wild type flies $+200 \mu \mathrm{g} / \mathrm{ml} \mathrm{C} 1+$ Paraquat

4. Group IV: Wild type flies $+200 \mu \mathrm{g} / \mathrm{ml} \mathrm{C} 2+$ Paraquat

5. Group V: Wild type flies $+200 \mu \mathrm{g} / \mathrm{ml} \mathrm{C} 4+$ Paraquat

6. Group VI: Wild type flies $+200 \mu \mathrm{g} / \mathrm{ml}$ EDA + Paraquat 


\section{Paraquat resistance test}

Two days after emergence from the pupae, as per the grouping, 50 male flies were fed with the standard food with $1 \%$ DMSO in the control group. Flies in the treatment groups were fed with the food containing test compounds $\mathrm{C} 1, \mathrm{C} 2$, $\mathrm{C} 4$, and EDA respectively for seven days. Flies from all the groups along with paraquat group except control group were starved for $6 \mathrm{~h}$ to ensure that no food remained in the digestive tract so that none of the compounds would alter the uptake of paraquat. Later, these flies were transferred to vials containing only filter paper soaked with $15 \mathrm{mM}$ paraquat in a $5 \%$ sucrose solution. The survival of the flies was determined $24 \mathrm{~h}$ and $48 \mathrm{~h}$ after the paraquat exposure. Survived flies were used for homogenization for biochemical assays. Each assay was repeated thrice. ${ }^{17,18}$

\section{Biochemical investigations}

Whole-body homogenates of flies were prepared in 0.1 M Sodium-phosphate buffer (pH7.4) after performing the study. 30 male flies from the batch I and II were used for this purpose. After homogenizing, the samples were centrifuged. The supernatant was filtered through a nylon mesh (pore size, $10 \mu \mathrm{m}$ ) and used for biochemical assays. ${ }^{17}$

\section{Assay for Lipid Peroxidation}

Lipid peroxidation was measured by employing thiobarbituric acid (TBA). Absorption was measured at $532 \mathrm{~nm}$ against the blank and malondialdehyde (MDA) equivalents were measured from the calibration curve of MDA. ${ }^{19}$ The results were expressed as nMol malondialdehyde per mg protein.

\section{Assay for Hydroperoxide}

Hydroperoxide generation was determined in the homogenate of flies using FOX reagent. The absorbance was taken at $560 \mathrm{~nm}$. Hydrogen peroxide content was estimated from the calibration curve of hydroperoxide and results were expressed as nMol HP/mg protein. ${ }^{20}$

\section{Estimation of Reduced Glutathione}

Reduced glutathione (GSH) content was estimated based on the reported method. The reduced glutathione was estimated from the calibration curve of reduced glutathione standard and the results were expressed as mg GSH per mg protein. $^{21}$

\section{Antioxidant enzyme assays}

Catalase activity in the homogenate was estimated through standard reported methods. The results were expressed as $\mu \mathrm{Mol}$ hydrogen peroxide per mg protein. SOD activity was monitored for $3 \mathrm{~min}$ at $406 \mathrm{~nm}$, expressed as the amount of protein required to inhibit $50 \%$ of quercetin auto-oxidation. ${ }^{22,23}$

\section{Locomotor assay}

The locomotor capacity was evaluated by following the negative geotaxis behaviour of drosophila flies of different groups as mentioned in the study design described by Coulom and Birmanwith some modifications. Twenty flies per group were anaesthetized and placed in a vertical glass column (length, $25 \mathrm{~cm}$; diameter, $1.5 \mathrm{~cm}$ ) sealed at one end. After a brief recovery period, flies were gently tapped to the bottom of the column. Following $1 \mathrm{~min}$, the number of flies that reached $6 \mathrm{~cm}$ of the column (passed) and flies that remained below this mark (failed) were noted. The assays were repeated three times for each fly. Results were represented as the percentage of flies passed the test (mean $\pm \mathrm{SD}$ ) obtained from three independent experiments. ${ }^{17,18}$

\section{Statistical analysis}

Statistical analysis was performed using one-way analysis of variance (ANOVA) followed by Dunnet's test. The values were expressed as Mean \pm S.D computed from all the three replicates. Prism software (Ver. 5.0) and Microsoft Excel was used for statistical analysis.

\section{RESULTS}

\section{Structures of compounds}

The synthesized compounds formed were recrystallized and analytically characterized through FTIR, ${ }^{1} \mathrm{H}$ NMR, ${ }^{13} \mathrm{C}$ NMR, and LCMS. The data is reported previously. ${ }^{10,11}$

\section{In vitro antioxidant studies}

\section{DPPH radical scavenging activity}

All the compounds showed moderate to very good antioxidant activity in comparison with standard EDA. The compound C4 exhibited good DPPH scavenging activity than EDA. Compounds $\mathrm{C} 1$ and $\mathrm{C} 2$ showed less activity than $\mathrm{C} 4$. The percentage of inhibition data is depicted in figure $1 \mathrm{~A}$. $\mathrm{IC}_{50}$ values for all the compounds were calculated. $\mathrm{IC}_{50}$ for $\mathrm{C} 4$ is significant in comparison with the standard data is illustrated in table 2 .

\section{ABTS radical scavenging assay and total antioxidant capacity assay}

All the compounds were screened for ABTS and total antioxidant capacity assay. Among the tested compounds, C4 showed good activity which is significant compared to EDA. All other compounds showed moderate antioxidant activity. The data is given in figures $1 \mathrm{~B}$ and $1 \mathrm{C}$ respectively.

\section{Ferric reducing antioxidant power assay}

Compounds were assayed for ferric reducing antioxidant power assay and data are expressed as ascorbic acid equiva- 
lents. Among the compounds, $\mathrm{C} 4$ showed significant activity in comparison with EDA. The compound $\mathrm{C} 1$ showed the least activity among the tested compounds. The data is illustrated in figure 1D.

\section{Neuroprotection studies in Drosophila flies}

\section{Lethality and Paraquat exposure studies}

PQ induced lethality test was conducted to determine the nonlethal concentration of compounds. The mortality rate of flies treated with $10,15,20$, and $25 \mathrm{mM}$ concentration of PQ for $96 \mathrm{~h}$ in the interval of $24 \mathrm{~h}$ is depicted in figure 2A. Exposure of adult flies to PQ resulted in a concentration-dependent lethality during a $96 \mathrm{~h}$ experimental period. Mortality occurred between $72 \mathrm{~h}$ and $96 \mathrm{~h}$ among paraquat exposed flies. The cumulative percent mortality at the tested concentrations is shown in figure 2A. From the results obtained, $15 \mathrm{mM}$ concentration of PQ is selected for further studies. Flies were pretreated with compounds at different concentrations viz., 50,100 , and $200 \mu \mathrm{g} / \mathrm{ml}$. Lethality was determined by counting dead flies every $24 \mathrm{~h}$ for 7 days. The data were compared with normal media-fed flies. Compounds were found to be safe under-tested concentrations.

\section{Paraquatresistance studies}

The flies were pretreated with the compoundsC1, C2, C4, and EDR for 7 days. Compounds pretreated D. melanogaster showed increased survival rate after paraquat exposure in contrast with the controls; the difference was significant ( $p$ $<0.001$ ) in comparison with EDA pretreated flies with C4. Other compounds showed moderate protective ability. Data are expressed as survival percentage and reported in figure 2B.

\section{Locomotor assay (Negative geotaxis assay)}

Paraquat induced neurotoxicity was evidenced through locomotor deficits in normal flies fed with common media containing PQ. Flies with locomotor deficits prefer to remain at the bottom of the glass column and do not appear to coordinate their legs in a normal fashion. Compound $\mathrm{C} 4$ was able to substantially save the flies from worsening locomotive dysfunctions in comparison with EDA pretreated and compound unfed flies.C1 and C2 pretreated flies showed moderate activity in comparison with $\mathrm{C}$. Data are illustrated in figure 3.

\section{In vivo antioxidant and oxidative stress marker assays}

Lipid peroxidation Malondialdehyde (MDA) and Hydroperoxide (HP)

There was a significant elevation in the level of malondialdehyde (MDA) and hydroperoxide (HP) in the case of PQ treated flies. C1, C2, C4, and EDA supplemented diet for 7 days showed a significant diminution in MDA (Figure4A) and HP levels (Figure4B). The endogenous levels of oxidative stress markers (MDA and HP) were reduced significantly in $\mathrm{C} 4$ pretreated flies concerning EDA pretreated flies. Compound $\mathrm{C} 4$ showed good antioxidant activity in comparison with $\mathrm{C} 1$ and $\mathrm{C} 2$.

\section{Reduced Glutathione}

The levels of reduced GSH were significantly enhanced in whole-body homogenates among flies fed with compounds. Among the compounds, $\mathrm{C} 4$ was successful in ameliorating the activity of GSH in the flies when compared with EDA pretreated ones. In the case of PQ pretreated flies, the GSH level was decreased significantly in comparison with normal flies. Compound $\mathrm{C} 4$ was also successful in elevating GSH levels in comparison with PQ pretreated flies. $\mathrm{C} 1$ and $\mathrm{C} 2$ pretreated flies showed moderate activity. The data is illustrated in figure $5 \mathrm{~A}$.

\section{Activities of Antioxidant Enzymes CAT and SOD}

Compounds pretreated flies showed moderate activity to restore antioxidant enzyme levels viz., superoxide dismutase (Figure 5B), and catalase (Figure 5C). Among the tested compounds, $\mathrm{C} 4$ showed significant activity in comparison with EDA pretreated flies. However, these compounds were not successful in bringing SOD and CAT levels to basal levels in comparison with normal flies.

\section{DISCUSSION}

Oxidative stress is induced by an imbalanced redox state, involving the either excessive generation of reactive oxygen species (ROS) or dysfunction of the antioxidant system, thereby leading to neurodegenerative diseases like Alzheimer's and Parkinson's. Antioxidant therapy has been suggested for the prevention and treatment of neurodegenerative diseases. ${ }^{24}$ The capacity of antioxidants to nullify the oxidative stress-mediated damage is supposed to contribute to their therapeutic potential in preventing or slowing down neurodegeneration. Several studies have demonstrated that the antioxidant compounds protect neuronal cells by neutralizing the excessive free radicals and/or by enhancing the antioxidant defences. ${ }^{25,26}$

For elucidating the neuroprotective activity, we synthesized the pyrazolone derivatives (reported) which are structurally identical with standard drug Edaravone. It is a member of the substituted 2-pyrazolin-5-one and IUPAC name is 3-methyl1-phenyl-2-pyrazolin-5-one. It was originally developed as a potent free radical scavenger and neuroprotective agent in several diseases in vivo, including stroke, spinal cord injury, traumatic brain injury, neurodegenerative diseases, and brain tumors. ${ }^{27,28}$ Due to its antioxidant and neuroprotective effi- 
cacy, Edaravone was selected as a standard compound for in vivo antioxidant and neuroprotective studies.

The compounds were evaluated for in vitro antioxidant property through DPPH scavenging, ABTS assay, ferric reducing power ability and total antioxidant capacity assay. The compounds showed good to moderate antioxidant activity. Among all the test compounds, $\mathrm{C} 4$ exhibited good in vitro antioxidant activity. The good activity of the compound might be due to methyl substitution on 5-Pyrazolone. It was reported that methyl substitution enhanced antioxidant efficacy in pyrazolone. Our data is well correlated with previously reported work. ${ }^{29}$

Further, the selected compounds were evaluated for in vivo antioxidant and neuroprotective studies in Drosophila melanogaster OK flies. Oxidative stress plays an important role in governing the life span of the fly. ${ }^{30}$

The antioxidant ability of the synthetic compounds was estimated by inducing oxidative stress in the flies using paraquat (1,1-dimethyl-4-4-bipyridinium dichloride). It is a quaternary nitrogen herbicide and highly toxic substance. The toxicity of paraquat is due to the generation of the superoxide anion which can lead to the synthesis of toxic reactive oxygen species (ROS) such as hydroxyl radicals, hydrogen peroxide and leads to the cellular apoptosis. ${ }^{31}$ In this study, we observed the lethality of $D$. melanogaster due to paraquat treatment as reported earlier. However, the significant reduction in mortality rate with the compound treatment in PQ exposed flies indicates the protective effect of the compounds. This might be due to their free radical scavenging activity which neutralizes ROS generated by paraquat.

Further, pretreatment of flies with compounds C1, C2 and $\mathrm{C} 4$, exhibited in vitro antioxidant property. The dose of compounds for pretreatment was determined through toxicity studies (study design was explained in methods). In this experiment, $1 \%$ Dimethyl sulfoxide (DMSO) was taken as an aprotic solvent that can solubilize a wide variety of poorly soluble polar and nonpolar molecules. This is coupled with its apparent low toxicity at concentrations $<10 \%$ which has led to its ubiquitous use and widespread application..$^{32}$ Our results confirmed no effect of solvent on drosophila at tested doses comparable to the normal flies.

Antioxidant efficacy of the compounds was determined by estimating the oxidative stress markers and antioxidant enzymes in the whole-body homogenate of flies. Paraquat exposed flies showed significant induction of oxidative stress as evidenced by the elevation in the levels of MDA, HP, and depletion of GSH. This supports the fact that it is a potent oxidative stress inducer, greatly increases the ROS production, and inhibits the regeneration of reducing equivalents, compounds necessary for the antioxidant activity. It was observed that there was a statistically significant reduction in the levels of MDA and HP in C4 pre-treated flies. Other compounds showed moderate but insignificant activity. The level of GSH was significantly elevated in case of C4compound treated flies. $\mathrm{C} 4$ was successful in modulating the activity of GSH by bringing the levels to near basal, which could be attributed to lipid peroxidation inhibition capacity of the compound. The results were analyzed statistically and found significant at the $\mathrm{P}<0.05$ level.

The antioxidant enzymes such as SOD and CAT levels were induced by PQ in the fly which was upturned in compound fed flies. The compound $\mathrm{C} 4$ was successful in modulating the activities by bringing antioxidant enzymes SOD and CAT to near basal levels comparable with normal flies by restoring redox condition. In paraquat sublethal dose exposed flies, locomotor defects were noted due to the selective degeneration of dopaminergic neurons. Drosophila is being widely used as a model to understand the mechanisms involved in neurodegenerative disorders, to model environmental toxininduced parkinsonism and a convenient system to screen therapeutic agents for their neuroprotective effects, before their testing in mammalian model. ${ }^{33}$ It is known that nigrostriatal dopaminergic neurons are very sensitive to the toxicity of paraquat. ${ }^{34,35}$ Our results showed that flies exposed to PQ exhibited locomotion deficits and stayed at the bottom of the jar in the negative geotaxis assay, as they could not coordinate their legs while climbing. The flies pretreated with C4 compounds before PQ exposure showed significant improvement in locomotor activity. Other compounds showed less activity in comparison with $\mathrm{C} 4$.

In general, a pyrazolone molecule containing labile hydrogen as $-\mathrm{NH}-\mathrm{C}=\mathrm{O}$ group in the ring exhibit the tautomerism phenomenon. This makes the molecule to act as a good antioxidant scaffold. But the compounds $\mathrm{C} 1$ and $\mathrm{C} 2$ have similar pyrazolone moiety without a tautomerism phenomenon. This could be due to the presence of an intramolecular hydrogen bond formed between the oxygen atom of the carbonyl group of pyrazolone ring and the hydrogen atom attached to the nitrogen of exocyclic azo group. The formed N-H---$\mathrm{O}=\mathrm{C}$ bond hinders the tautomeric phenomenon owing to the formation of a six-membered ring, which might be giving stability to the system. It is worth noting that a report has been cited for the existence of arylhydrazonotautomer, both in solid form as well as in solution for a similar analogue. ${ }^{36-}$ ${ }^{38}$ This might be the reason for the poor antioxidant activity of the compounds $\mathrm{C} 1$ and $\mathrm{C} 2$. This kind of bonding was not observed in compound $\mathrm{C} 4$ and edaravone molecule. Hence, they showed potent antioxidant activity.

This is the study that first reported on the neuroprotective potential of tested compounds in the Drosophila model against PQ induced oxidative stress. This work contributes a new scaffold towards the development of a potential neuroprotective agent. Further investigations are required to unravel the 
mechanisms of its action at the cellular and higher organism levels.

\section{CONCLUSION}

Our results suggest that pyrazolone derivative prophylaxis has the propensity to protect against neurotoxic exposure might be due to its antioxidant potential. In this model, the protective activity of $\mathrm{C} 4$ was comparable with EDA known neuroprotective agent. Our data further confirm the utility value of the drosophila system as a primary model to rapidly screen potential compounds for their antioxidant and neuroprotection properties before their testing in mammalian models.

\section{ACKNOWLEDGEMENT}

Authors acknowledge Dr. B K Sarojini, Professor, and Head, Department of Industrial Chemistry, Mangalore University for her constant support in the synthesis and characterization of the compounds.

\section{Conflict of interest}

The authors declare that there are no conflicts of interest.

\section{Source of Funding: Nil}

\section{REFERENCES}

1. Bondy SC. The relation of oxidative stress and hyperexcitation to neurological disease. Proc Soc Exp Biol Med. 1995; 208:33745.

2. Indika BG, Nicholas A. Medical management of paraquat ingestion. Br J Clin Pharmacol 2011; 72: 745-57.

3. Suntres ZE. Role of antioxidants in paraquat toxicity.Toxicol 2002; 180:65-77.

4. Shimizu K, Matsubara K, Ohtaki K, Fujimaru S, Saito O, et al. Paraquat induces long-lasting dopamine overflow through the excitotoxic pathway in the striatum of freely moving rats. Brain Res 2003; 976:243-52.

5. Ying H, Heinrich J. Studying ageing in Drosophila. Meth 2014;68: 129-33.

6. Jayaraj RL, Tamilselvam K, Manivasagam T, Elangovan N. Neuroprotective effect of CNB-001, a novel pyrazole derivative of curcumin on biochemical and apoptotic markers against rotenone-induced SK-N-SH cellular model of Parkinson's disease. J Mol Neurosci 2013; 51:863-70.

7. Abdel-Aziz M, Abuo-Rahma Gel D, Hassan AA. Synthesis of novel pyrazole derivatives and evaluation of their antidepressant and anticonvulsant activities. Eur J Med Chem 2009; 44:3480-7.

8. Murozono M, Kanazawa M, Nara T. Edaravone and cyclosporine A as neuroprotective agents for acute ischemic stroke. Acute Med Surg 2018; 5: 213-21.

9. Cruz MP. A Novel neuroprotective agent for the treatment of amyotrophic lateral sclerosis. Pharm Ther 2018;43: 25-8.

10. Thomas YR, Mary S, Resmi KS. Synthesis and spectroscopic study of two new pyrazole derivatives with the detailed compu- tational evaluation of their reactivity and pharmaceutical potential. J Mol Struc 2019; 1181: 599-612.

11. Mohan BJ, Sarojini BK, Narayana B, Sindhu Priya ES. (Z)4(3,4Difluorophenyl)hydrazono]-3-methyl-1Hpyrazol-5(4H)one. Molbank 2018; M989.

12. Barros L, Baptista P, Isabel CFR. Effect of Lactarius piperatus fruiting body maturity stage on antioxidant activity measured by several biochemical assays. Food ChemToxicol 2007; 45: 1731-7.

13. Alam MN, Bristi NJ, Rafiquzzaman M. Review on in vivo and in vitro methods evaluation of the antioxidant activity. Saudi Pharm J 2013; 21: 143-52.

14. Prieto P, Pineda M, Aguilar M. Spectrophotometric quantitation of antioxidant capacity through the formation of a phosphomolybdenum complex: specific application to the determination of Vitamin E. Anal Biochem 1999; 269: 337-41.

15. Farvin KHS, Andersen LL, Nielsen HH. Antioxidant activity of Cod (Gadusmorhua) protein hydrolysates: In vitro assays and evaluation in 5\% fish oil-in-water emulsion.Food Chem 2014; 149:326- 34.

16. Ravikumar H, Muralidhara. Neuroprotective efficacy of $B a-$ copa monnieri against rotenone induced oxidative stress and neurotoxicity in Drosophila melanogaster. Neurotoxicol 2009; 30:977-85.

17. Darshan Raj CG, Sarojini BK, Ramakrishna MK. Protective effect of bischalcone derivative in Drosophila melanogaster against electron beam radiation. Res Pharm 2011; 1:38-43.

18. CoulomH, Birman S. Chronic exposure to rotenone models sporadic Parkinson's disease in Drosophila melanogaster. J Neurosci 2004; 48: 10993-8.

19. Ohakawa H, Ohishi U, Yakgi K.Assay for lipid peroxides in animal tissues by thiobarbituric acid reaction. Anal Biochem 1979; 95:145-9.

20. Wolf SP. Ferrous ion oxidation in presence of ferric ion indicator xylenol orange for measurement of hydroperoxides. Methods Enzymol 1994; 233: 182-9.

21. Thomas WS, Siraj JI, Richard AL. Selenium modulates peroxidation in the absence of glutathione peroxidase in Musca domestica. Biochem Biophys Res Commun. 1989; 165: 15863.

22. Mahmoud HH, Hussein NA. Data supporting the spectrophotometric method for the estimation of catalase activity. Data in Brief 2016; 6: 194-9.

23. Kakkar BD, Viswanathan PN. A modified spectrophotometric assay of superoxide dismutase. Ind J Biochem Biophys 1984; 21:130-2.

24. Geon HK, Jieun EK, Sandy JR, Sujung Y. The role of oxidative stress in neurodegenerative diseases. Exp Neurobiol 2015; 24 : 325-40.

25. Ishige K, Schubert D, Sagara Y. Flavonoids protect neuronal cells from oxidative stress by three distinct mechanisms. Free Rad Biol Med 2001; 30: 433-46.

26. Kanski J, Aksenova M, Stoyanova A, Butterfield DA. Ferulic acid antioxidant protection against hydroxyl and peroxyl radical oxidation in synaptosomal and neuronal cell culture systems in vitro: Structure-activity studies. J Nutr Biochem 2002; 13: 27381.

27. Kikuchi K, Kawahara K, Tancharoen S. The free radical scavenger edaravone rescues rats from cerebral infarction by attenuating the release of high-mobility group box-1 in neuronal cells. J Pharmacol Exp Ther 2009;329:865-74.

28. Kikuchi K, Tancharoen S, Matsuda F. Edaravone attenuates cerebral ischemic injury by suppressing aquaporin-4. BiochemBiophys Res Commun 2009;390:1121-5. 
29. Kiyoshi F, Ikuo N, Atsuko M, Tomohiro M, Sachiko H, Mikiko $\mathrm{H}$, et al. Effect of methyl substitution on the antioxidative property and genotoxicity of resveratrol.Chem ResToxicol 2008;21:282-7.

30. Orr WC, Radyuk SN, Prabhudesai L, Toroser D, Benes JJ, Luchak JM, et al.Overexpression of glutamate-cysteine ligase extends life span in Drosophila melanogaster. J Biol Chem 2005;280:37331-8.

31. Rodrigo F, Sumin L. Humberto RR, Michaela B, Mihalis IP. Molecular mechanisms of pesticide-induced neurotoxicity: Relevance to Parkinson's disease. Chemicobiol Int 2010;188:289300 .

32. Joana G, Benjamin D, Mark T, Eduardo N, Michael RD, CordeiroMF.Unexpected low-dose toxicity of the universal solvent DMSO. FASEB J 2020;1317.

33. Pienaar IS, Go"tz J, Feany MB. Parkinson's disease:insights from non-traditional model organisms. Progress Neurobiol 2010;92:558-71.

Table I: Structures of pyrazolone derivatives used in this study

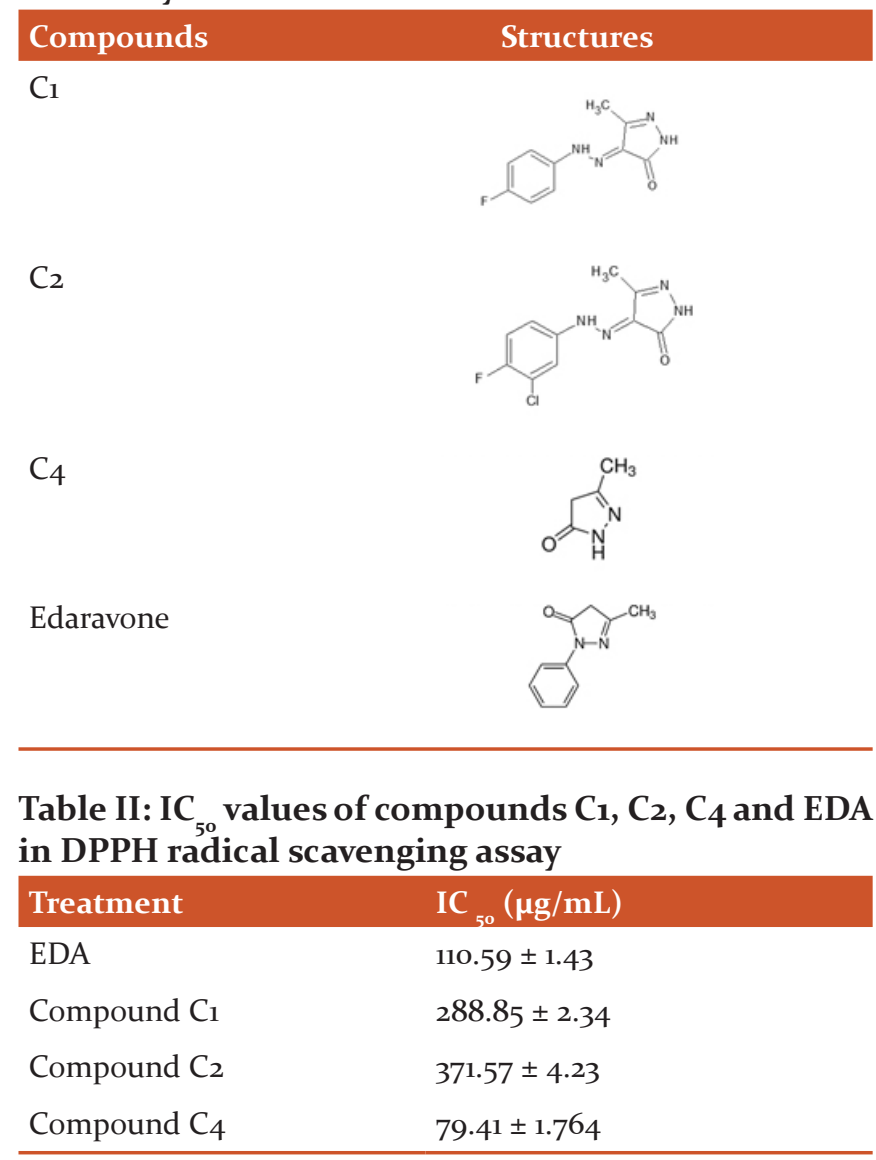

34. Thiruchelvam M, McCormack A, Richfield EK, Baggs RB, Tank AW, Di Monte DA, Cory-Slechta DA. Agerelated irreversible progressive nigrostriatal dopaminergic neurotoxicity in the paraquat and maneb model of the Parkinson's disease phenotype. Eur J Neurosci 2003; 18:589-600.

35. McCormack AL, Atienza JG, Johnston LC, Andersen JK, Vu S, Di Monte DA. Role of oxidative stress in paraquat-induced dopaminergic cell degeneration. J Neurochem 2005; 93:1030-7.

36. Fun HK, Quah CK, Nithinchandra KB. 4-[2- (4- Chlorophenyl) hydrazinylidene]-3-methyl-1H-pyrazol-5(4H)-one. Acta Cryst E 2011; E67: o2670.

37. Khalil AK, Hassan MA, Mohamed MM, El-Sayed AM. Metal salt-catalyzed diazocoupling of 3-substituted-1H-pyrazol-2-in5-ones in aqueous medium. Dyes Pigment 2005; 66: 241-5.

38. Hassan AEA, Moustafa AH, Tolbah MM, Zohdy HF, Haikal AZ. Synthesis and antimicrobial evaluationofnovel Pyrazolones and Pyrazolonenucleosides. Nucleosides Nucleotides Nucleic Acids 2012; 31: 783-800.

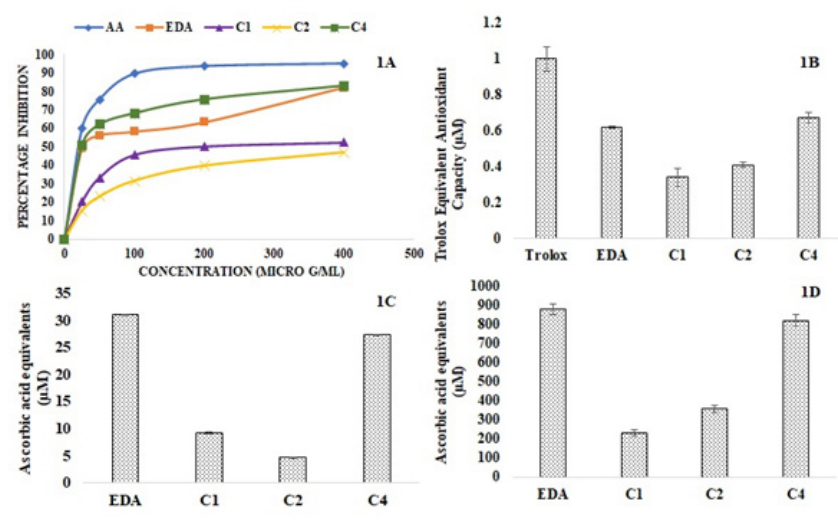

Figure 1: Represents invitro antioxidant assays such as $1 \mathrm{~A}$ : DPPH scavenging activity; 1B: ABTS assay; 1C: TAC assay; 1D: FRAP assay of compounds C1, C2, C4 and EDA. Values were presented as Mean \pm SD.

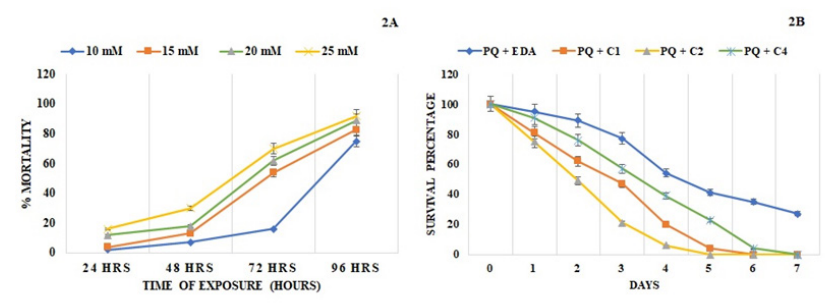

Figure 2: $A: P Q$ mortality test; $B$ : Paraquat co-exposure survival study. Values were expressed as Mean \pm SD (in triplicates). 


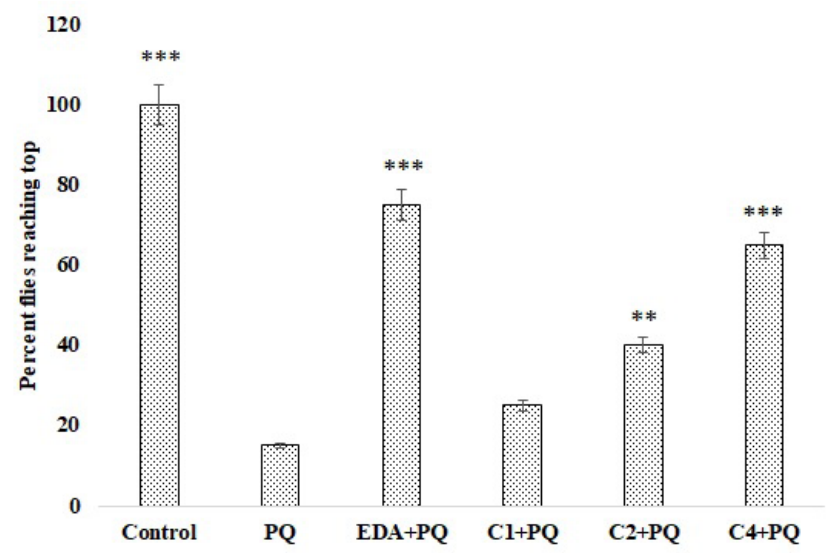

Figure 3: Modulation of paraquat-induced locomotor (expressed as percent flies escaped) deficits among adult male Drosophila melanogaster by compounds and standard EDA treatments ( $n=50$ flies per replicate, three such replications used for assay). Values were expressed as Mean $\pm S D ;{ }^{* * *} p<0.001,{ }^{* *} p<0.01$ compared to $P Q$.

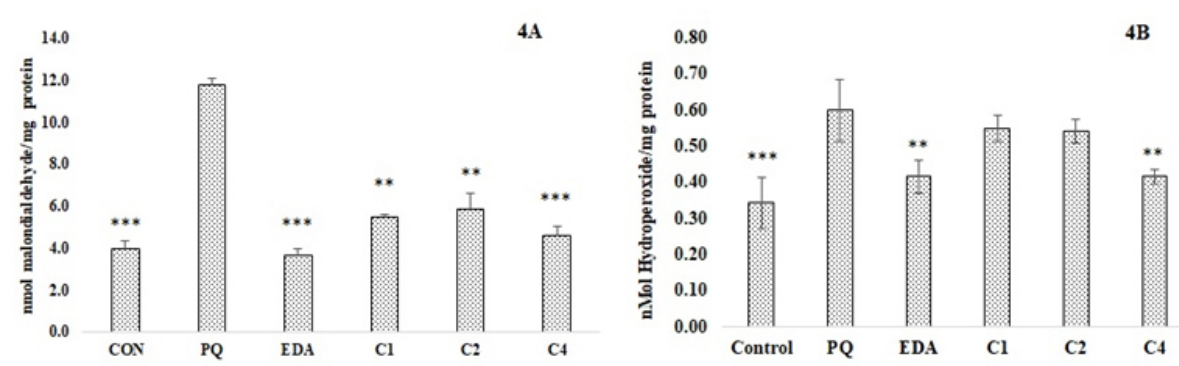

Figure 4: $A$ : Modulatory effect of $C 1, C 2, C 4$ and EDA treatments on paraquat $(P Q)$ induced oxidative stress measured as malondialdehyde levels, B: Hydroperoxide in whole-body homogenates of adult $D$. melanogaster. Values were expressed as Mean $\pm \mathrm{SD} ;{ }^{* * *} \mathrm{p}<0.001,{ }^{* *} \mathrm{p}<0.01$ compared to $\mathrm{PQ}$.

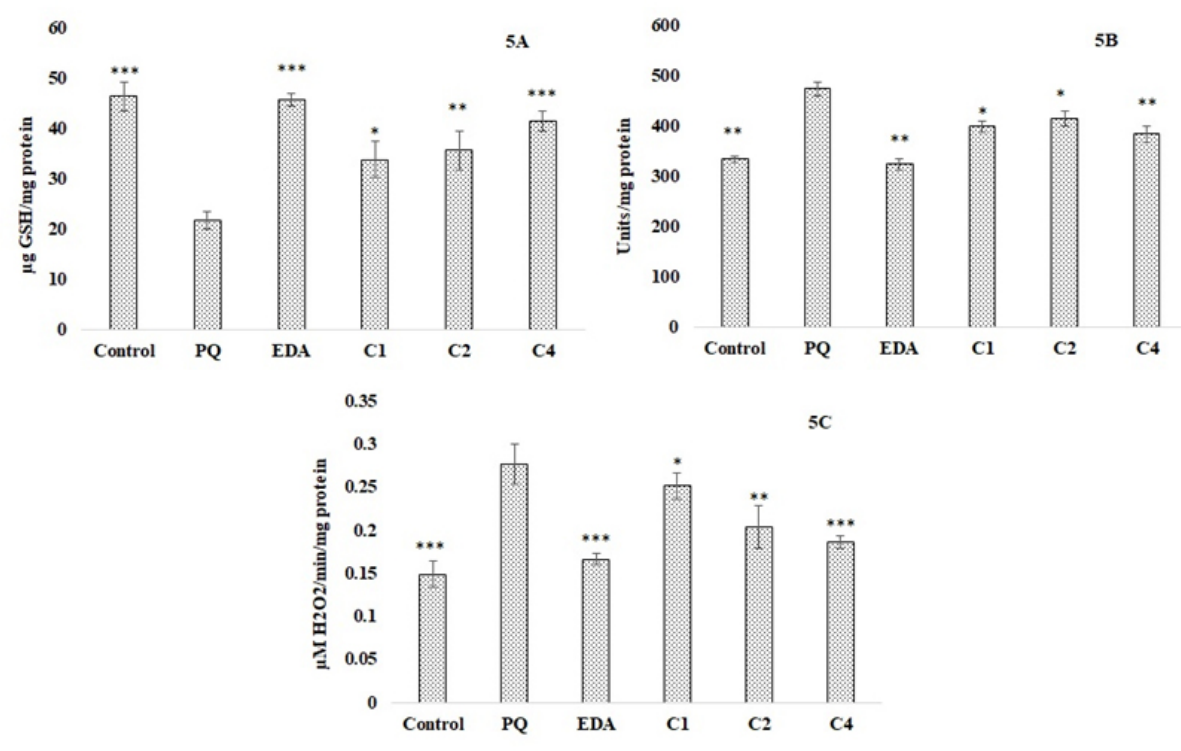

Figure 5: A: Ameliorative effect of $\mathrm{C} 1, \mathrm{C} 2$, and $\mathrm{C} 4$ treatments on $\mathrm{PQ}$ induced oxidative stress measured as reduced glutathione levels, B: SOD; C: CAT assay in whole-body homogenates of adult $D$. melanogaster. Values were expressed as Mean \pm SD; ${ }^{\star * *}$ $p<0.001,{ }^{* *} p<0.01,{ }^{*} p<0.05$ compared to $P Q$. 